\title{
ARTICLE
}

\section{Lipidic cubic phase injector facilitates membrane protein serial femtosecond crystallography}

Uwe Weierstall', Daniel James ${ }^{1}$, Chong Wang ${ }^{2}$, Thomas A. White ${ }^{3}$, Dingjie Wang ${ }^{1}$, Wei Liu ${ }^{2}$, John C.H. Spence ${ }^{1}$, R. Bruce Doak1, Garrett Nelson ${ }^{1}$, Petra Fromme ${ }^{4}$, Raimund Fromme ${ }^{4}$, Ingo Grotjohann ${ }^{4}$, Christopher Kupitz ${ }^{4}$, Nadia A. Zatsepin1, Haiguang Liu', Shibom Basu", Daniel Wacker², Gye Won Han², Vsevolod Katritch², Sébastien Boutet ${ }^{5}$, Marc Messerschmidt ${ }^{5}$, Garth J. Williams ${ }^{5}$, Jason E. Koglin ${ }^{5}$, M. Marvin Seibert ${ }^{5,6}$, Markus Klinker $^{3,7}$, Cornelius Gati ${ }^{3}$, Robert L. Shoeman ${ }^{8}$, Anton Barty ${ }^{3}$, Henry N. Chapman 3,9,10, Richard A. Kirian 1,3, Kenneth R. Beyerlein ${ }^{3}$, Raymond C. Stevens ${ }^{2}$, Dianfan Li ${ }^{11}$, Syed T.A. Shah ${ }^{11}$, Nicole Howe ${ }^{11}$, Martin Caffrey ${ }^{11}$ \& Vadim Cherezov $^{2}$

Lipidic cubic phase (LCP) crystallization has proven successful for high-resolution structure determination of challenging membrane proteins. Here we present a technique for extruding gel-like LCP with embedded membrane protein microcrystals, providing a continuously renewed source of material for serial femtosecond crystallography. Data collected from sub$10-\mu \mathrm{m}$-sized crystals produced with less than $0.5 \mathrm{mg}$ of purified protein yield structural insights regarding cyclopamine binding to the Smoothened receptor.

\footnotetext{
${ }^{1}$ Department of Physics, Arizona State University, Tempe, Arizona 85287, USA. ${ }^{2}$ The Scripps Research Institute, Department of Integrative Structural and Computational Biology, La Jolla, California 92037, USA. ${ }^{3}$ Center for Free-Electron Laser Science, DESY, Notkestrasse 85, Hamburg 22607, Germany. ${ }^{4}$ Department of Chemistry and Biochemistry, Arizona State University, Tempe, Arizona 85287, USA. ${ }^{5}$ SLAC National Accelerator Laboratory, 2575 Sand Hill Road, Menlo Park, California 94025, USA. ${ }^{6}$ Laboratory of Molecular Biophysics, Department of Cell and Molecular Biology, Uppsala University, Husargatan 3 (Box 596), Uppsala SE-751 24, Sweden. ${ }^{7}$ Departamento de Química, Universidad Autónoma de Madrid, Ciudad Universitaria de Cantoblanco, Madrid 28049, Spain. ${ }^{8}$ Max-Planck-Institut für medizinische Forschung, Jahnstrasse 29, Heidelberg 69120, Germany. ${ }^{9}$ Department of Physics, University of Hamburg, Hamburg 22761, Germany. ${ }^{10}$ Center for Ultrafast Imaging, Hamburg 22607, Germany. ${ }^{11}$ School of Medicine and School of Biochemistry and Immunology, Trinity College Dublin, Dublin 2, Ireland. Correspondence and requests for materials should be addressed to U.W. (email: weier@asu.edu) or to V.C. (email: vcherezo@scripps.edu).
} 
$\mathrm{M}$ embrane proteins constitute about one third of the proteome in most organisms, perform critical cellular and physiological functions and represent over $60 \%$ of current drug targets in humans ${ }^{1}$. High-resolution threedimensional structures of membrane proteins are indispensable for understanding their functional mechanisms and designing novel drugs with high selectivity and potency. However, knowledge of membrane protein structures lags behind that of soluble proteins ${ }^{2}$, emphasizing the need to develop innovative methods and approaches.

Beginning with the seminal work on photosynthetic reaction centers ${ }^{3}$, membrane proteins have historically been crystallized in detergent micelle solutions. About 17 years ago, an alternative method of crystallization was introduced, based on the use of a membrane-mimetic medium known as the lipidic cubic phase $(\mathrm{LCP})^{4,5}$. This technique has proven crucial for determining highresolution structures and functional mechanisms of membrane proteins from several diverse families, starting with microbial rhodopsins ${ }^{6}$ and including $G$ protein-coupled receptors, ion channels, transporters and enzymes ${ }^{7-11}$.

While LCP crystallization typically produces highly ordered crystals, these crystals are often limited in size. A high density of micrometer-sized crystals in the LCP is often obtained during initial screening, but subsequent optimization to obtain sufficiently large crystals for data collection at synchrotron sources can be laborious and time consuming. Despite the fact that microcrystallography has matured over the last few years ${ }^{5,12}$, structure determination of membrane proteins using microcrystals remains difficult. Ultimately, the achievable resolution for well-ordered small crystals is limited by radiation damage ${ }^{13}$ that poses an inherent problem for all conventional $\mathrm{X}$-ray-based methods of structure determination.

LCP-grown microcrystals are ideally suited for the emerging technique of serial femtosecond crystallography (SFX) ${ }^{14,15}$. SFX relies on the fact that the duration of the $\mathrm{X}$-ray pulses generated by an X-ray free-electron laser (XFEL) is so brief $(<50 \mathrm{fs})$, that diffracted photons exit the sample before damage initiated by photoionization can establish itself. Diffraction is thereby recorded from essentially undamaged molecules at or close to room temperature. The peak brightness of an XFEL is a billion times higher than that of third generation synchrotrons, allowing collection of high quality single diffraction patterns from individual sub- $10-\mu \mathrm{m}$-sized crystals in random orientations. After collecting several hundred thousand of such patterns at a rapid rate, structure factors are determined by Monte Carlo-type integration over the measured diffraction intensities ${ }^{16}$. The first experimental demonstrations of SFX, at low resolution, were carried out with membrane proteins crystallized in detergent solution ${ }^{17}$ and in the liquid-like lipidic sponge phase ${ }^{18}$. Recently, the first structures of soluble proteins in aqueous dispersion have been solved at atomic resolution ${ }^{19,20}$.

To date, the SFX method has been based on X-ray data collection from a liquid stream containing protein micro/ nanocrystals. The gas dynamic virtual nozzle (GDVN), which is used to inject microcrystals in their mother liquor into the X-ray beam, produces a liquid jet flowing at $10 \mathrm{~m} \mathrm{~s}^{-1}$ and focused to 1$5 \mu \mathrm{m}$ diameter by employing shear and pressure forces from a coflowing ga $^{21}$. Hence, given the $120 \mathrm{~Hz}$ X-ray pulse repetition rate of the Linac Coherent Light Source (LCLS), the sample stream advances several centimeters between X-ray pulses, which are focused to $0.1-2 \mu \mathrm{m}$ diameter. Consequently, in a typical SFX experiment, only 1 out of 10,000 microcrystals is probed by the $\mathrm{X}$-ray beam. With a liquid flow rate of $10 \mu \mathrm{min}^{-1}$, it takes 5-6h to collect a full data set, thus requiring $10-100 \mathrm{mg}$ of pure protein. Obtaining such amounts is not feasible for many membrane proteins.

Because of its gel-like nature, LCP allows operation at much lower stream speeds and more efficient sample utilization. Its high viscosity, however, makes it incompatible with GDVN techniques. A new approach was needed to generate a micrometer-sized stream of LCP suitable for SFX.

We report here the development of a novel method and a device for extruding LCP at slow flow speeds and with extremely low sample consumption as a continuous $10-50 \mu \mathrm{m}$ diameter stream. It provides a continuously renewed sample target for interrogation by the femtosecond X-ray beam. The flow speed of the injector is adjustable to the X-ray pulse repetition rate of the XFEL, so that no sample is wasted between shots. LCP-grown microcrystals of the human smoothened (SMO) receptor in complex with cyclopamine have been injected into the femtosecond X-ray beam. Diffraction data from 61,964 microcrystals were merged to recover the structure to $3.2 / 4.0 \AA$ resolution. The protein consumption is reduced by a factor of 20 compared with SFX experiments with the GDVN nozzle.

\section{Results}

LCP injection. The LCP microextrusion injector (Fig. 1) consists of a hydraulic stage, a sample reservoir and a nozzle. The reservoir can hold up to $20 \mu \mathrm{l}$ of LCP and is connected to a fused silica capillary with $10-50 \mu \mathrm{m}$ inner diameter. The LCP is extruded out of this capillary into an evacuated sample chamber and requires a pressure of 2,000-10,000 psi, depending on the nozzle diameter and flow speed. This is provided by the hydraulic stage that amplifies the applied pressure by a factor of 34 . Shear force exerted by a co-flowing gas (helium or nitrogen

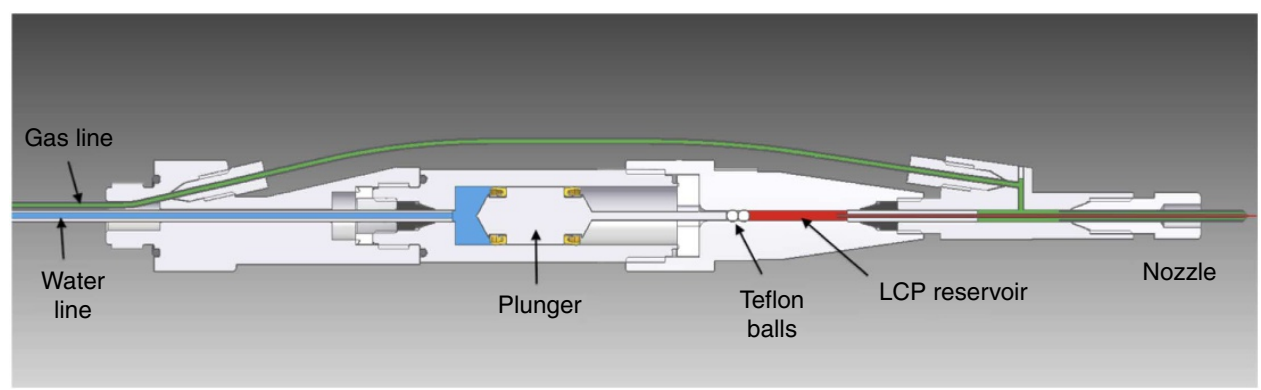

Figure 1 | Middle section through the LCP injector. In operation, the device is attached via the leftmost threaded fitting to a nozzle rod (not shown) for insertion into the experimental chamber. Water (blue) and gas (green) lines are routed through the nozzle rod from the left. LCP (red) is extruded from the nozzle on the right. Water, at a pressure of up to 300 psi, drives the hydraulic plunger, which amplifies the pressure 34 times to drive LCP through a capillary with an inner diameter of $10-50 \mu \mathrm{m}$. Two spherical Teflon beads are used to provide a tight seal against a pressure of up to 10,000 psi. The co-flowing gas is necessary for reliable extrusion and to maintain co-axial flow. 
at $300-500$ psi supply pressure) keeps the LCP stream on axis (see Methods for details).

The LCP flow rate (typically $1-300 \mathrm{nl} \mathrm{min}^{-1}$ ) depends on the sample composition, nozzle diameter and pressure and can be optimized for the $120 \mathrm{~Hz}$ pulse rate of the LCLS, so that between $\mathrm{X}$-ray pulses, the stream advances only the distance needed to expose fresh sample to the next pulse, dramatically reducing sample consumption compared with GDVN injection (Supplementary Movie 1).

The most commonly used lipid for crystallization of membrane proteins in LCP is monoolein, 9.9 MAG (an N.T MAG shorthand notation is used for monounsaturated monoacylglycerol lipids, where ' $\mathrm{N}$ ' is the number of carbon atoms in the acyl chain between the ester and cis-olefinic bonds, and ' $\mathrm{T}$ ' is the number of carbon atoms between the cis-olefinic bond and the end of the chain). However, this lipid is not ideally suited for the LCP-SFX experiments, as it undergoes a phase transition from the cubic phase to a lamellar crystalline $\left(\mathrm{L}_{\mathrm{c}}\right)$ phase at $18^{\circ} \mathrm{C}$. As $\mathrm{LCP}$ is injected into an evacuated sample chamber at $\sim 10^{-3}$ Torr and $20^{\circ} \mathrm{C}$, evaporative cooling can transform it into the $\mathrm{L}_{\mathrm{c}}$ phase, leading to strong, sharp diffraction rings from the $\mathrm{L}_{c}$ phase (Fig. 2a). This dramatically increases the background and poses a danger to
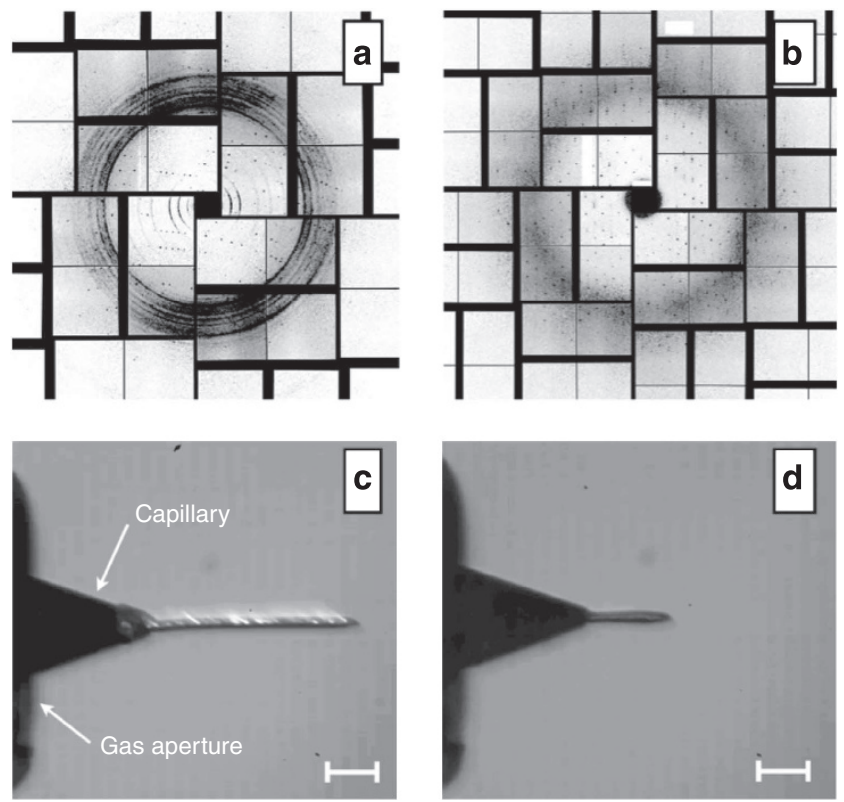

Figure 2 | Snapshot diffraction patterns and LCP extrusion. (a,b) Single femtosecond snapshot diffraction patterns. (a) Diffraction spots from $A_{2 A}$ adenosine receptor microcrystals in $9.9 \mathrm{MAG} /$ cholesterol LCP to $2.5 \AA$ and strong powder diffraction rings from crystalline lipid. (X-ray intensity attenuated to $7 \%, 1.5 \mu \mathrm{m}$ X-ray beam diameter, 50 -fs pulse length, $9.5 \mathrm{keV}$,

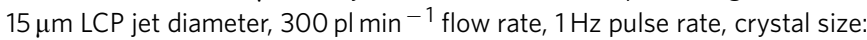
$1 \times 1 \times 5 \mu \mathrm{m}^{3}$ ). (b) Diffraction from serotonin receptor $5-\mathrm{HT}_{2 \mathrm{~B}}$ in cholesterol-doped 9.9 MAG + 7.9 MAG LCP. No sharp rings are visible suggesting that formation of $L_{c}$ phase has been avoided ( $X$-ray intensity attenuated to $3.1 \%$ due to strong Bragg diffraction from $5 \times 5 \times 5 \mu \mathrm{m}^{3}$ sized crystals, $1.5 \mu \mathrm{m}$ X-ray beam diameter, 50 -fs pulse length, $9.5 \mathrm{keV}, 50 \mu \mathrm{m}$ LCP jet diameter, $190 \mathrm{nl} \mathrm{min}^{-1}$ flow rate, $120 \mathrm{~Hz}$ pulse rate). The resolution at the detector edge in both panels is $2.5 \AA$. Panels (c) and (d): 9.9 MAG LCP extrusion in vacuum viewed between crossed polarizers. The tapered end of the capillary nozzle is seen protruding out of the gas aperture. Capillary inner diameter: $30 \mu \mathrm{m}$. (c) with $\mathrm{He}$ as co-flowing gas. Birefringence (bright flecks) is an indication of a transition of the cubic phase to a lamellar crystalline phase due to evaporative cooling. (d) with $\mathrm{N}_{2}$ as co-flowing gas and no visible birefringence. Scale bars, $100 \mu \mathrm{m}$. the detector, which made it necessary to attenuate the LCLS beam by a factor of 20-30. Nonetheless, in our initial experiments both microcrystal samples of $\beta_{2}$ adrenergic receptor $\left(\beta_{2} A R\right)$ and adenosine $\mathrm{A}_{2 \mathrm{~A}}$ receptor $\mathrm{A}_{2 \mathrm{~A}} \mathrm{AR}$ showed consistent diffraction to $\sim 2.5 \AA$, even with the highly attenuated beam (Fig. $2 \mathrm{a}$ ).

By changing the co-flowing gas from $\mathrm{He}$ to $\mathrm{N}_{2}$, the formation of the $\mathrm{L}_{c}$ phase was suppressed but not completely eliminated in the case of LCP prepared with 9.9 MAG (Fig. 2c,d). However, by replacing the 9.9 MAG with shorter chain MAGs $\left(7.9 \mathrm{MAG}^{22}\right.$ or 9.7 MAG (monopalmitolein) available from Avanti Polar Lipids), the formation of the $\mathrm{L}_{\mathrm{c}}$ phase was completely prevented. Diffraction patterns collected at the LCLS confirmed the presence of the expected cubic-Pn3m phase, without a trace of the $\mathrm{L}_{\mathrm{c}}$ phase (Fig. 2b). In addition, we have established that for crystals that only grow in 9.9 MAG LCP (the most successful crystallization host lipid to date), 7.9 MAG can be added post crystal growth to prevent formation of the $\mathrm{L}_{c}$ phase upon injection (see Methods for details), which greatly expands the range of proteins amenable to this method. The high quality of the X-ray diffraction data collected from crystals grown in 9.9 MAG that were delivered in the 7.9/9.9 MAG mixture (Fig. 2b) show that the crystals do not suffer from the addition of 7.9 MAG.

Diffraction Data and Sample Consumption. Using the LCP injector with a flow rate of $170 \mathrm{nl} \mathrm{min}^{-1}$, SFX data were acquired for several $G$ protein-coupled receptors, including $\beta_{2} A R, A_{2 A} A R$, $\mathrm{SMO}$, glucagon receptor and serotonin $2 \mathrm{~B}\left(5-\mathrm{HT}_{2 \mathrm{~B}}\right)$ receptor ${ }^{23}$, as well as the membrane enzyme diacylglycerol kinase (DgkA). Full data sets for SFX structure determination were collected for DgKA, SMO and $5-\mathrm{HT}_{2 \mathrm{~B}}$ over the course of $5-10 \mathrm{~h}$, while using less than $100 \mu \mathrm{l}$ of each sample $(<0.5 \mathrm{mg}$ of protein). This is a vast improvement over typical sample consumption with a GDVN nozzle, which requires $10 \mathrm{ml}$ (10 $\mathrm{mg}$ protein) for a complete data set ${ }^{17}$. A comparison of the amounts of sample used in different experiments with the GDVN and the LCP injector is included in Supplementary Table 1.

As proof of principle for the newly developed LCP-SFX method, we analysed the data collected on human SMO receptor in complex with the naturally occurring teratogen cyclopamine. SMO belongs to the class Frizzled of $G$ protein-coupled receptor superfamily, and participates in embryonic development and tumour growth. The first SMO structure in complex with an antagonist LY2940680 was recently determined by traditional microcrystallography at a synchrotron source ${ }^{24}$. We were, however, unsuccessful in solving the structure of the SMO/ cyclopamine complex using synchrotron data collected with a $10 \mu \mathrm{m}$ diameter X-ray beam due to poor diffraction from relatively large crystals $\left(\sim 120 \times 10 \times 5 \mu \mathrm{m}^{3}\right.$; Supplementary Fig. 1c,d), which presumably suffered from accumulation of crystal growth defects or from effects related to cryocooling. The LCP-SFX data collected on sub-5 $\mu$ m-sized crystals (Supplementary Fig. 1a,b) at room temperature were of a reasonable quality to solve the structure by molecular replacement after application of an anisotropic data truncation at 3.4, 3.2 and $4.0 \AA$ along three principal crystal axes (Table 1). Although the resolution is not very high, it does allow us to confidently locate where this small molecule ligand with an antitumor therapeutic potential ${ }^{25}$ binds (Fig. 3). This crystallographic model will be further refined by subsequent biochemical studies.

\section{Discussion}

In summary, our successful development of an LCP microextrusion injector allows the beneficial attributes of SFX measurements 
to be coupled with the unique properties and advantages of LCP for membrane protein crystallization. The new technology not only enables the collection of high-resolution structure data from LCP-grown membrane protein microcrystals at room temperature, but also dramatically reduces the amount of protein required, eliminates the need for laborious crystal size optimization and simplifies crystal handling procedures.

\section{Table 1 | Data collection and refinement statistics.}

\begin{tabular}{|c|c|}
\hline & SMO/cyclopamine \\
\hline \multicolumn{2}{|l|}{ Data collection } \\
\hline Space group & $\mathrm{P} 2_{1}$ \\
\hline \multicolumn{2}{|l|}{ Cell dimensions, } \\
\hline$a, b, c(\AA)$ & $40.5,157.3,52.4$ \\
\hline$\alpha \beta \gamma\left(^{\circ}\right)$ & $90.0,97.0,90.0$ \\
\hline Resolution $(\AA)$ & $40-3.20(3.26-3.20)^{\star}$ \\
\hline$R_{\text {split, }}(\%)$ & $9.8(63.2)$ \\
\hline$I / \sigma(I)$ & $7.4(1.8)$ \\
\hline$C C^{\star}$ & $0.9991(0.28)$ \\
\hline Completeness (\%) & $100(100)$ \\
\hline Multiplicity & $2,515(1,820)$ \\
\hline \multicolumn{2}{|l|}{ Refinement } \\
\hline Resolution ( $\AA)$ & $40-3.20$ \\
\hline Anisotropic truncation $(\AA)$ & $3.4,3.2,4.0$ \\
\hline No. reflections/test set & $8,082 / 399$ \\
\hline$R_{\text {work }} / R_{\text {free }}$ & $0.232 / 0.278$ \\
\hline \multicolumn{2}{|l|}{ No. atoms } \\
\hline Protein & 3,389 \\
\hline Cyclopamine & 30 \\
\hline \multicolumn{2}{|l|}{$B$-factors $\left(\AA^{2}\right)$} \\
\hline Wilson B/overall B & $101.6 / 78.4$ \\
\hline Receptor/BRIL & $76.8 / 83.7$ \\
\hline Cyclopamine & 76.3 \\
\hline \multicolumn{2}{|l|}{ R.m.s. deviations } \\
\hline Bonds lengths $(\AA)$ & 0.003 \\
\hline Bond angles $\left({ }^{\circ}\right)$ & 0.61 \\
\hline
\end{tabular}

\section{Methods}

LCP Injector. The LCP injector, consisting of a hydraulic stage, a sample reservoir and a nozzle, is mounted onto the end of the nozzle rod of an injector designed for XFEL ${ }^{26}$. The nozzle rod can be removed from the vacuum chamber without compromising the vacuum, allowing for sample reloading and nozzle change. The hydraulic stage consists of a syringe body with a sealed solid plunger. The syringe body has a large bore on the inlet side and a much smaller bore on the outlet side. The ratio of the respective bore areas gives a nominal amplification factor of 34, delivering $10,200 \mathrm{psi}$ to the LCP reservoir when water on the inlet side is pressurized to $300 \mathrm{psi}$.

The large bore in the syringe body has a diameter of $8 \mathrm{~mm}$. The plunger sliding in this bore has two groves that accept high pressure hydraulic seals (Trelleborg Turcon Variseal) and has a diameter of $8 \mathrm{~mm}$ on the water side and an extension with a diameter of $1.37 \mathrm{~mm}$ on the LCP side. The plunger extension drives two Teflon beads (Bal-tec) of $1.59 \mathrm{~mm}$ outer diameter, which slide in the precision bored $1.37 \mathrm{~mm}$ diameter LCP reservoir bore (Fig. 1). The nominal pressure amplification (neglecting frictional forces on the seals) is 34 , the ratio of the squared diameters of the bores $(8 \mathrm{~mm})^{2}(1.37 \mathrm{~mm})^{-2}$. The Teflon beads seal the bore of the reservoir against LCP leakage. When the beads are pressed into the slightly smaller reservoir bore, they deform cylindrically. The pressure applied by the plunger deforms them further and thus provides a tight seal for pressures up to $10,000 \mathrm{psi}$ on the LCP.

The $360 \mu \mathrm{m}$ outer diameter and $10-50 \mu \mathrm{m}$ inner diameter fused silica capillary tubing (Polymicro) is kept as short as possible $(6 \mathrm{~cm})$ to reduce the pressure necessary to extrude the LCP. The nozzle section and the capillary are connected to the reservoir via standard HPLC conical ports (Upchurch with 10-32 threads). The tapered end of the inner capillary is inserted into a flame-polished square outer glass tube and protrudes out of the exit aperture, so that gas can flow through the open corners at a rate adequate for LCP extrusion (for details of nozzle design, see Weierstall et $\mathrm{l}^{26}{ }^{26}$. The reservoir bore is filled with LCP via a Hamilton syringe and can hold a volume of $20 \mu \mathrm{l}$. The Teflon beads are replaced during each sample refill. It is possible to drive the LCP flow in a 'constant pressure' mode via pressurized water connected to the plunger inlet. The supply reservoir for the water is, in turn, pressurized by gas from a gas cylinder and the LCP flow rate is controlled by adjusting the gas pressure. However, in this mode of operation the LCP flow rate would occasionally show sudden jumps (possibly due to wall stick-slip ${ }^{27}$ of the LCP), leading to sample loss. Better control over LCP flow was achieved in a 'constant flow rate' mode implemented by using a HPLC pump (Shimadzu LC$20 \mathrm{AD}$ ) to drive the hydraulic liquid (water).

The LCP flow rate can be optimized for the $120 \mathrm{~Hz}$ pulse rate of the LCLS so that between X-ray pulses, the stream advances only the distance needed to expose fresh sample to the next pulse (Supplementary Movie 1). The necessary distance (the 'damage diameter') depends on the X-ray beam diameter and pulse energy. Thus, for example, at an X-ray energy of $9.5 \mathrm{keV}$, a pulse energy of $50 \mu \mathrm{J}$ at the sample and a beam diameter of $1.5 \mu \mathrm{m}$, this distance is $10-30 \mu \mathrm{m}$. Consequently, for a flow speed where the LCP stream travels $10-30 \mu \mathrm{m}$ between X-ray pulses $\left(1.2-3.6 \mathrm{~mm} \mathrm{~s}^{-1}\right)$, little, if any, sample is wasted and sample consumption is reduced dramatically compared with GDVN injection. Constant LCP flow rates of

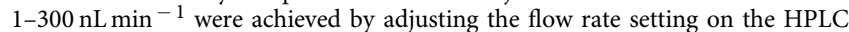
pump or by using constant pressure mode (for the lowest flow rates). The minimum usable flow rate is set by the diameter of the LCP stream, the distance
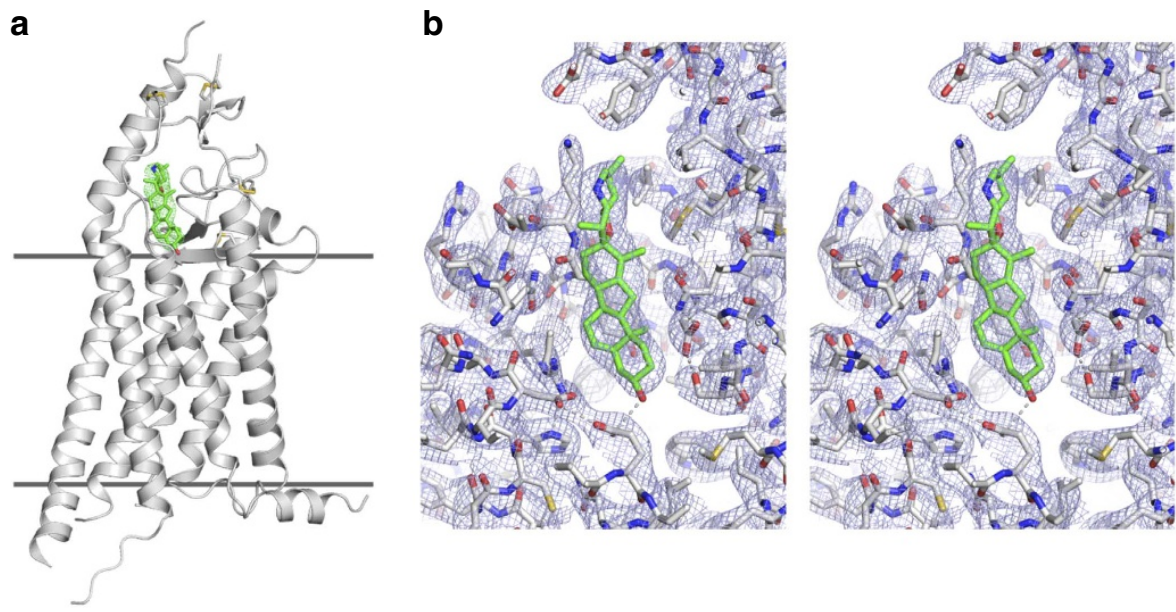

Figure 3 | Cyclopamine binding to smoothened receptor. (a) Receptor model is shown as a grey cartoon, cyclopamine as a stick model with green carbons, and the 'omit' $2 \mathrm{mF}_{\mathrm{o}}-\mathrm{DF}_{\mathrm{c}}$ density map for cyclopamine contoured at $1 \sigma$ is shown as green wires. Horizontal lines indicate membrane boundaries. (b) Cyclopamine binding pocket is shown in stereo view as a stick model along with $2 \mathrm{mF}_{\mathrm{o}}-\mathrm{DF}_{\mathrm{c}}$ density map contoured at $1 \sigma$. Cyclopamine binds near to the entrance into a long and narrow cavity inside the receptor. Polar interactions stabilizing the shape of the cavity and cyclopamine binding are shown as grey dashed lines. Viewing angles in (a) and (b) are slightly different. 
that the stream must advance between XFEL pulses, and the X-ray pulse repetition rate (for example, for $120 \mathrm{~Hz}$ repetition rate, LCP stream diameter of $15 \mu \mathrm{m}$ and damage diameter of $20 \mu \mathrm{m}$, the minimum usable flow rate is $25 \mathrm{nl} \mathrm{min}^{-1}$ ). The LCP flow rate can be calculated by dividing the HPLC pump flow rate by the pressure amplification factor 34 .

GPCR Expression and Purification. Purified GPCR samples $\left(\beta_{2} \mathrm{AR}, \mathrm{A}_{2 \mathrm{~A}} \mathrm{AR}\right.$, glucagon receptor and 5- $\left.\mathrm{HT}_{2 \mathrm{~B}}\right)^{24,28-31}$ were prepared as follows. Constructs engineered for crystallization were expressed in Sf9 insect cells for $48 \mathrm{~h}$ at $27^{\circ} \mathrm{C}$ using recombinant baculovirus. Cells were harvested and total membranes were purified by repeated Dounce homogenization and centrifugation in hypotonic and hypertonic buffer. GPCR-ligand complexes were subsequently formed by incubating purified membranes in the presence of ligand, followed by extraction of the complexes in $1 \%(\mathrm{w} / \mathrm{v}) \mathrm{N}$-dodecyl- $\beta$-D-maltopyranoside (Anatrace) and $0.2 \%(\mathrm{w} / \mathrm{v})$ cholesteryl hemisuccinate (CHS, Sigma). Solubilized proteins were purified by immobilized metal affinity chromatography and concentrated to $\sim 20-50 \mathrm{mg} \mathrm{ml}^{-1}$.

GPCR Crystallization. Purified protein was reconstituted into LCP by mixing protein solution with molten monoolein $(9.9 \mathrm{MAG}) / 10 \%(\mathrm{w} / \mathrm{w})$ cholesterol at a volume ratio $2 / 3$ (protein:lipid) using a syringe mixer $^{32}$. Initial crystallization screening was performed using an NT8-LCP robot (Formulatrix) dispensing $40 \mathrm{nl}$ of protein-laden LCP and overlaying it with $800 \mathrm{nl}$ precipitant solution in each well of a 96-well glass sandwich plate (Marienfeld). Plates were stored at $20^{\circ} \mathrm{C}$ and periodically imaged in an incubator/imager RockImager 1000 (Formulatrix). Several conditions producing high density of small crystals within $24 \mathrm{~h}$ were selected for scaling up (Supplementary Fig. 1a,b).

Crystals for LCP-SFX were obtained in Hamilton gas-tight syringes by injecting $\sim 5 \mu$ of protein-laden LCP as a continuous column of $\sim 400 \mu \mathrm{m}$ in diameter into a $100 \mu \mathrm{l}$ syringe filled with $60 \mu \mathrm{l}$ of precipitant solution and incubated for at least $24 \mathrm{~h}$ at $20^{\circ} \mathrm{C}$. After crystal formation and removal of excess precipitant solution, $\sim 3 \mu \mathrm{l}$ of molten 7.9 MAG lipid ${ }^{22}$ were added to absorb the residual precipitant solution and prevent formation of $\mathrm{L}_{\mathrm{c}}$ phase upon injection of LCP into vacuum. The resulting crystal containing LCP sample $(\sim 10 \mu \mathrm{l})$ was inspected under visual and ultraviolet microscopes as well as SONICC ${ }^{33}$ at the LCLS and loaded in the LCP injector for LCP-SFX data collection. Samples with high crystal density were further diluted two times with LCP prepared from 1:1 ratio of 7.9 MAG and corresponding precipitant solution.

Smoothened Receptor. An engineered human smoothened receptor construct with truncated amino-terminal cysteine-rich domain (residues 1-189) and carboxy terminus at Q555, and a thermostabilized Escherichia coli apocytochrome B562 $\left(\right.$ BRIL $^{34}$ fused into intracellular loop 3 (replacing residues from P434 to K440) was generated, expressed and purified as described in the GPCR expression and purification section. The $\Delta$ CRD-SMO-BRIL- $\Delta C$ construct in complex with cyclopamine readily produced high-density microcrystals in LCP following the crystallization protocol described in the GPCR crystallization section using a variety of precipitant conditions, including $100 \mathrm{mM}$ Hepes $\mathrm{pH} 7.0,30 \%(\mathrm{v} / \mathrm{v})$ PEG 400, $100 \mathrm{mM}$ salt (Supplementary Fig. 1a,b). After several rounds of optimization, relatively large crystals obtained in conditions with ammonium salts (Supplementary Fig. 1c,d) were used for data collection at a synchrotron source, and microcrystals obtained in $100 \mathrm{mM}$ Hepes pH 7.0, 30\% (v/v) PEG 400, $100 \mathrm{mM}$ $\mathrm{NaCl}$ were used for LCP-SFX data collection.

DgkA Expression and Purification. The coding sequence of a thermostable mutant variant of DgkA, with its N-terminal methionine replaced by a hexa-His tag-containing decapeptide (MGHHHHHHEL) to facilitate purification, was synthesized and cloned ${ }^{10}$. This mutant, referred to as $\Delta 7$ DgkA, incorporated seven mutations with respect to wild-type DgkA, as follows: A41C, C46A, I53V, I70L, M96L, V107D and C113A. DgkA production and purification, primarily from inclusion bodies, were carried out following published procedures ${ }^{10}$.

DgkA Crystallization. Crystallization trials began with the reconstitution of the protein into the bi-layer of the lipidic cubic mesophase following a standard protocol ${ }^{32}$. The protein solution at $12 \mathrm{mg} \mathrm{ml}^{-1}$ was homogenized with $7.9 \mathrm{MAG}$ in equal parts by volume using a coupled syringe device at room temperature $\left(20-22^{\circ} \mathrm{C}\right)$. Approximately $20 \mu \mathrm{l}$ of protein-laden mesophase was transferred into a $0.5 \mathrm{ml}$ Hamilton syringe containing $0.4 \mathrm{ml}$ precipitant solution $(0.2 \%(\mathrm{v} / \mathrm{v}) \mathrm{MPD}$, $0.1 \mathrm{M}$ sodium chloride, $0.1 \mathrm{M}$ sodium citrate $\mathrm{pH}$ 5.6) using a narrow bore coupler $^{32}$, as described above for GPCR crystallization. The syringe was incubated for 21 days at $20^{\circ} \mathrm{C}$ for crystal growth. After separating the bathing solution from the crystal-laden LCP, excess precipitant was absorbed by mixing in 3-5 $\mu$ l molten 7.9 MAG. This procedure produced optically clear LCP in which microcrystals were dispersed ready for loading into the reservoir of the LCP injector, as described above.

Experimental Setup at LCLS and Data Acquisition Rates. The experiment took place at the LCLS in the sample chamber of the Coherent X-ray Imaging instrument ${ }^{35}$, using $9.5 \mathrm{keV}(1.3 \AA) \mathrm{X}$-ray pulses with a pulse length of $50 \mathrm{fs}$ and repetition rate of $1-120 \mathrm{~Hz}$. The pressure in the experimental chamber was $\sim 10^{-5}$ Torr; the pressure at the extruded LCP sample was about $10^{-1}-10^{-3}$ Torr depending on the gas flow rate of the sheath gas. The nozzle area is pumped by a differential pumping system, which reduces gas flow from the nozzle into the main chamber ${ }^{26}$. Due to evaporative cooling, the sample temperature at the point of the $\mathrm{X}$-ray interaction ( $100 \mu \mathrm{m}$ downstream of the nozzle exit) is estimated to be slightly below room temperature. An XFEL beam diameter of $1.5 \mu \mathrm{m}$ was used. With an LCLS frequency of $60-120 \mathrm{~Hz}$ (depending on the LCP flow rate), one million diffraction patterns, which is a sufficiently large data set to achieve atomic resolution, can be collected in $\sim 2.5 \mathrm{~h}$ with $25 \mu \mathrm{l}$ of sample.

LCP-SFX data for SMO/cyclopamine crystals were collected at an X-ray pulserepetition rate of $120 \mathrm{~Hz}$. The sample-to-detector distance was $100 \mathrm{~mm}$ and the beam was attenuated to $3-6 \%\left(2.5-510^{10}\right.$ photons per pulse) of the full intensity to avoid detector saturation. A total of 3,510,525 diffraction patterns were collected, of which 274,214 were identified as potential single crystal diffraction patterns with more than 15 potential Bragg peaks by the software Cheetah ${ }^{36}$, corresponding to an average hit rate of $7.8 \%$. Auto indexing and structure factor integration of the crystal hits was performed using CrystFEL (version 0.5 .1$)^{37}$, resulting in 61,964 indexed images with a monoclinic lattice ( $22.6 \%$ indexing success rate). Based on the observation of systematic absences (Supplementary Fig. 2) and the behaviour of the Pearson correlation coefficient (Supplementary Fig. 3), we concluded that the diffraction data reach about $3.2 \AA$ resolution, but are highly anisotropic. The UCLA anisotropy server (http://www.services.mbi.ucla.edu/anisoscale/) was used to truncate data at $3.4,3.2$ and $4.0 \AA$ resolution along the three principal axes. Data collection statistics are summarized in Table 1 .

Structure determination and refinement. The SMO/cyclopamine structure was determined by molecular replacement with the program Phaser $^{38}$ using the receptor domain of the previously reported SMO structure in complex with LY2940680 (PDB ID: 4JKV) and the BRIL domain from 5- $\mathrm{HT}_{2 \mathrm{~B}}$ structure (PDB ID: 4IB4) as the search models. After several rounds of alternate refinement by Phenix.refine ${ }^{39}$, including simulated annealing to remove phase bias, and manual inspection and corrections in $\operatorname{Coot}^{40}$ in the absence of any ligand, extra electron density was observed in the same vicinity of the LY2940680 ligand binding site (Fig. 3). Due to the limited diffraction resolution and a relatively featureless shape of cyclopamine, orienting this ligand in the available elongated density was not straightforward and required combining additional information based on chemical knowledge to create a reasonable model. Since it has been reported that cyclopamine derivatives with bulky substitution groups attached to its secondary amine retain their ability to bind $\mathrm{SMO}^{25,41}$, cyclopamine was placed in the density with its secondary amine group pointing to the open extracellular space needed to accommodate bulky substitutions. After aligning the major axis of cyclopamine, two alternative orientations of cyclopamine differing by about $180^{\circ}$ rotation around its major axis could still be placed in the available electron density. One of these orientations was selected for further refinement based on the favourable conformer energy for cyclopamine, although the second orientation could not be entirely ruled out. The obtained model containing cyclopamine was further refined resulting in $R_{\text {work }} / R_{\text {free }}=0.232 / 0.278$ (Table 1$)$. The structure has an excellent geometry according to the MolProbity server ${ }^{42}$ with $95.6 \%$ residues in the favored and $4.4 \%$ residues in the allowed Ramachandran conformations. Residues 345-354 (intracellular loop 2) and 497-504 (extracellular loop 3) were not modelled due to poor density in these regions.

\section{References}

1. Yildirim, M. A., Goh, K.-I., Cusick, M. E., Barabási, A.-L. \& Vidal, M. Drug-target network. Nat. Biotechnol. 25, 1119-1126 (2007).

2. White, S. H. The progress of membrane protein structure determination. Protein Sci. 13, 1948-1949 (2004).

3. Deisenhofer, J., Epp, O., Miki, K., Huber, R. \& Michel, H. Structure of the protein subunits in the photosynthetic reaction centre of Rhodopseudomonas viridis at $3 \AA$ resolution. Nature 318, 618-624 (1985).

4. Cherezov, V. et al. Rastering strategy for screening and centring of microcrystal samples of human membrane proteins with a sub- $10 \mu \mathrm{m}$ size X-ray synchrotron beam. J. R. Soc. Interface 6, S587-S597 (2009).

5. Landau, E. M. \& Rosenbusch, J. P. Lipidic cubic phases: a novel concept for the crystallization of membrane proteins. Proc. Natl Acad. Sci. USA 93, 14532-14535 (1996).

6. Pebay-Peyroula, E. X-ray structure of Bacteriorhodopsin at 2.5 Angstroms from microcrystals grown in lipidic cubic phases. Science 277, 1676-1681 (1997).

7. Cherezov, V. et al. High-resolution crystal structure of an engineered human 2-adrenergic G protein coupled receptor. Science 318, 1258 (2007).

8. Santos, J. S. et al. Crystal structure of a voltage-gated $\mathrm{K}+$ channel pore module in a closed state in lipid membranes. J. Biol. Chem. 287, 43063-43070 (2012)

9. Liao, J. et al. Structural insight into the ion-exchange mechanism of the sodium/calcium exchanger. Science 335, 686-690 (2012).

10. Li, D. et al. Crystal structure of the integral membrane diacylglycerol kinase. Nature 497, 521-524 (2013). 
11. Cherezov, V. Lipidic cubic phase technologies for membrane protein structural studies. Curr. Opin. Struc. Biol. 21, 559-566 (2011).

12. Smith, J. L., Fischetti, R. F. \& Yamamoto, M. Micro-crystallography comes of age. Curr. Opin. Struc. Biol. 22, 602-612 (2012).

13. Garman, E. F. Radiation damage in macromolecular crystallography: what is it and why should we care? Acta Cryst. D 66, 339-351 (2010).

14. Spence, J., Weierstall, U. \& Chapman, H. X-ray lasers for structural and dynamic biology. Rep. Prog. Phys. 75, 102601 (2012).

15. Fromme, P. \& Spence, J. C. Femtosecond nanocrystallography using X-ray lasers for membrane protein structure determination. Curr. Opin. Struc. Biol. 21, 509-516 (2011).

16. Spence, J. C. H. et al. Phasing of coherent femtosecond X-ray diffraction from size-varying nanocrystals. Opt. Express. 19, 2866 (2011).

17. Chapman, H. N. et al. Femtosecond X-ray protein nanocrystallography. Nature 470, 73-77 (2011).

18. Johansson, L. C. et al. Lipidic phase membrane protein serial femtosecond crystallography. Nat. Methods 9, 263-265 (2012).

19. Boutet, S. et al. High-resolution protein structure determination by serial femtosecond crystallography. Science 337, 362-364 (2012).

20. Redecke, L. et al. Natively inhibited trypanosoma brucei cathepsin B structure determined by using an X-ray laser. Science 339, 227-230 (2013).

21. DePonte, D. P. et al. Gas dynamic virtual nozzle for generation of microscopic droplet streams. J. Phys. D. Appl. Phys. 41, 195505 (2008).

22. Misquitta, Y. et al. Rational design of lipid for membrane protein crystallization. J. Struct. Biol. 148, 169-175 (2004).

23. Liu, W. et al. Serial femtosecond crystallography of G protein-coupled receptors. Science 342, 1521-1524 (2013).

24. Wang, C. et al. Structure of the human smoothened receptor bound to an antitumour agent. Nature 497, 338-343 (2013).

25. Taipale, J. et al. Effects of oncogenic mutations in smoothened and patched can be reversed by cyclopamine. Nature 406, 1005-1009 (2000).

26. Weierstall, U., Spence, J. C. H. \& Doak, R. B. Injector for scattering measurements on fully solvated biospecies. Rev. Sci. Instrum. 83, 035108-035108 (2012).

27. Hatzikiriakos, S. G. Wall slip of molten polymers. Prog. Polym. Sci 37, 624-643 (2012).

28. Hanson, M. A. et al. A specific cholesterol binding site is established by the $2.8 \AA$ structure of the human $\beta 2$-adrenergic receptor. Structure. 16, 897-905 (2008).

29. Liu, W. et al. Structural basis for allosteric regulation of GPCRs by sodium ions. Science 337, 232-236 (2012).

30. Wacker, D. et al. Structural features for functional selectivity at serotonin receptors. Science 340, 615-619 (2013).

31. Siu, F. Y. et al. Structure of the human glucagon class B G-protein-coupled receptor. Nature 499, 444-449 (2013).

32. Caffrey, M. \& Cherezov, V. Crystallizing membrane proteins using lipidic mesophases. Nat. Protoc. 4, 706-731 (2009).

33. Kissick, D. J., Gualtieri, E. J., Simpson, G. J. \& Cherezov, V. Nonlinear optical imaging of integral membrane protein crystals in lipidic mesophases. Anal. Chem. 82, 491 (2010).

34. Chun, E. et al. Fusion partner toolchest for the stabilization and crystallization of G protein-coupled receptors. Structure. 20, 967-976 (2012).

35. Boutet, S. \& Williams, G. J. The coherent X-ray imaging (CXI) instrument at the linac coherent light source (LCLS). New J. Phys. 12, 035024 (2010).

36. White, T. A. et al. Crystallographic data processing for free-electron laser sources. Acta Cryst. D 69, 1231-1240 (2013).

37. White, T. A. et al. CrystFEL: a software suite for snapshot serial crystallography. I. Appl. Crystallogr. 45, 335-341 (2012).

38. Mccoy, A. J. et al. Phaser crystallographic software. J. Appl. Crystallogr. 40, 658-674 (2007)

39. Adams, P. D. et al. PHENIX: a comprehensive Python-based system for macromolecular structure solution. Acta Cryst. D 66, 213-221 (2010).

40. Emsley, P., Lohkamp, B., Scott, W. G. \& Cowtan, K. Features and development of Coot. Acta Cryst. D 66, 486-501 (2010).

41. Chen, J. K., Taipale, J., Cooper, M. K. \& Beachy, P. A. Inhibition of Hedgehog signaling by direct binding of cyclopamine to Smoothened. Genes Dev. 16, 2743-2748 (2002).
42. Chen, V. B. et al. MolProbity: all-atom structure validation for macromolecular crystallography. Acta Cryst. D 66, 12-21 (2009).

\section{Acknowledgements}

Experiments were carried out at the Linac Coherent Light Source, a national user facility operated by Stanford University on behalf of the U.S. Department of Energy (DOE), Office of Basic Energy Sciences. The work was supported in part by the National Institutes of Health Common Fund in Structural Biology grants P50 GM073197 (V.C. and R.C.S.), P50 GM073210 (M.C.), R01 GM095583 (P.F.) and NIGMS PSI:Biology grants U54 GM094618 (V.C. and R.C.S.), U54 GM094599 (P.F.), U54 GM094625 (M.C.) We acknowledge support from the National Science Foundation (awards MCB 1021557 and MCB 1120997) (J.C.H.S., U.W., P.F., R.B.D.) and its BioXFEL Science and Tech nology Center (NSF 1231306) and the Science Foundation Ireland (12/IA/1255). We further acknowledge support from the Helmholtz Gesellschaft and the Max Planck Gesellschaft (R.L.S.). Special thanks to T. Trinh and M. Chu for the help with baculovirus expression, F. Siu for help with sample preparation, and A. Walker for assistance with manuscript preparation. We are grateful to Richard Flubacher and the machinists of the ASU Mechanical Instrument Shop for their skill in fabricating injector components.

\section{Author contributions}

U.W. invented and designed the LCP injector and wrote the manuscript; operation of the sample delivery system was performed by U.W., D.J., Di.W., Ch.K., G.N.; W.L. developed microcrystal sample preparation in LCP protocol, prepared samples, assisted in testing the LCP injector, data collection and manuscript preparation; J.C.H.S. contributed to the development of the injector and the Monte Carlo method; R.B.D. contributed to the design of the LCP injector; P.F. was involved in the initiation and planning of the experiments, assisted with sample characterization, data collection and contributed to writing the paper; R.F., Ch. K., I.G. participated in data collection and contributed to sample characterization; N.A.Z., Sh.B., C.G. participated in data collection, processed and analysed data; H.L. helped with data analysis; Da.W. prepared GPCR microcrystals in LCP and helped with data collection; C.W. prepared SMO/cyclopamine microcrystals in LCP; T.A.W. analysed the SMO/cyclopamine data, G.W.H. refined the SMO/cyclopamine structure, V.K. analysed the structure and cyclopamine binding pose; Sé.B., M.M., G.J.W., J.E.K., M.M.S. setup the x-ray FEL experiment, beamline, controls and data acquisition, operated the Coherent X-ray Imaging beamline and performed the data collection; R.L.S. contributed to the design and operation of the LCP injector and delivery system, and read and revised manuscript; A.B. participated in data collection, wrote data processing software and helped with data processing and with writing the paper; H.N.C supervised software development and data processing, helped with writing the paper; R.A.K. developed the Monte Carlo integration method, contributed to data processing; K.R.B. and M.K. contributed to software development and data processing; R.C.S supervised GPCR production and contributed to writing the manuscript; D.L. and N.H produced and crystallized DgkA; D.L. helped with data collection; S.T.A.S. synthesized and purified 7.9 MAG; M.C. supervised the DgkA and 7.9 MAG synthesis and purification work, helped with data collection and with writing the paper; V.C. conceived the project, designed the experiments, supervised data collection, analysed the results and wrote the manuscript.

\section{Additional information}

Accession codes: The coordinates and structure factors of human smoothened receptor in complex with cyclopamine have been deposited in the Protein Data Bank under the accession code 4O9R.

Supplementary Information accompanies this paper at http://www.nature.com/ naturecommunications

Competing financial interests: The authors declare no competing financial interests.

Reprints and permission information is available online at http://npg.nature.com/ reprintsandpermissions/

How to cite this article: Weierstall, U. et al. Lipidic cubic phase injector facilitates membrane protein serial femtosecond crystallography. Nat. Commun. 5:3309 doi: 10.1038/ncomms4309 (2014). 